\title{
COMPLETE IDEALS IN LOCAL RINGS ${ }^{1}$
}

\section{H. T. MUHLY}

1. Let $L$ be a noetherian local ring with maximal ideal $M$. To each ideal $H$ of $L$ there corresponds an ideal $H_{a}$, the integral closure or $v$-completion of $H$, which consists of those elements $x$ in $L$ that depend integrally upon $H$ in the sense that an equation, $x^{n}+a_{1} x^{n-1}$ $+\cdots+a_{n}=0$, in which $a_{i} \in H^{i}, i=1,2, \cdots, n$, holds. Any ideal $H$ such that $H_{a}=H$ is called a $v$-complete ideal. It is well known [3] that $\left(H_{a}\right)_{a}=H_{a}$ for all ideals $H$. In this note we study the sequence $\left\{Q_{n}\right\}\left(Q_{n}=\left(M^{n}\right)_{a}\right)$ of the $v$-completions of the powers of the maximal ideal $M$ in the case where $L$ is a two dimensional local domain that is integrally closed in its quotient field $F$. Subject to certain finiteness restrictions explained below it is shown that there is an ideal $X$ generated by a set of parameters and an integer $s$ such that for each positive integer $t, Q_{s+t}=Q_{s} X^{t}$. By means of this result it is shown that the length $\lambda\left(Q_{n}\right)$ of the $M$-primary ideal $Q_{n}$ is given by a polynomial in $n$ when $n$ is large. Finally, it is shown that if $L$ is the local ring at a point $P$ of a normal algebraic surface $v$, if $u$ is the transform of $\mathcal{U}$ defined by a quadratic transformation with center $P$, and if $\mathcal{u}_{\delta}$ is a derived normal model of $u$ associated with the character of homogeneity $\delta$, then $\chi(\mathcal{V}, 2 n \delta)-\chi\left(\mathcal{U}_{\delta}, n\right)=\lambda\left(Q_{n \delta}\right)$, where $\chi$ is the Hilbert characteristic function. In particular, the constant term of the polynomial $\lambda\left(Q_{n}\right)$ measures the change in the relative arithmetic genus of $v$ that is induced by the quadratic transformation.

Some of the ideas and techniques used below are related to those employed by Rees in his study of pseudo-valuations [6, and related papers]. They go back ultimately, however, to Zariski's work on the reduction of singularities.

The author expresses his thanks to the referee for several helpful suggestions.

2. A subset $S$ of an ideal $H$ in a noetherian local ring $(L, M)$ will be called an $a$-basis of $H$ in case $H_{a}=(L S)_{a}$. The ideal $L S$ is then a reduction of $H$ in the sense of Northcott-Rees who have shown [5] that if $\left\{x_{1}, x_{2}, \cdots, x_{r}\right\}$ is a minimal $a$-basis of the ideal $M$, then the number $r$ of the elements $x_{i}$ is equal to the dimension of $L$, and

Presented to the Society, November 23, 1956 under the title On the relative arithmetic genus of a normal surface; received by the editors August 10, 1959.

1 Work on this paper was supported in part by a grant from the National Science Foundation. 
these elements are analytically independent. If $L$ is a domain integrally closed in its quotient field $F$ and if $\Omega$ is the set of valuations $v$ of $F$ such that $R_{v}$, the valuation ring of $v$, is an overring of $L$, and if $H_{b}=\{x ; v(x) \geqq v(H), \forall v \in \Omega\}$, then $H_{b}=H_{a}[3 ; 7]$. (Here $v(H)$ denotes $\min \{v(a) ; a \in H\}$.) Moreover, for each $v \in \Omega$ there is at least one $i$ such that $v\left(x_{i}\right)=v(M)$, so that $v\left(x_{i}^{n}\right)=v\left(M^{n}\right)$ for all integers $n \geqq 0$. Hence if $X=\sum\left(L x_{i}\right)$ is the ideal generated by the minimal $a$-basis $\left\{x_{1}, x_{2}, \cdots, x_{r}\right\}$, then $v\left(X^{n}\right)=v\left(M^{n}\right)$, and $Q_{n}=\left(X^{n}\right)_{b}$.

Let $L_{i}=L\left[x_{1} / x_{i}, x_{2} / x_{i}, \cdots, x_{r} / x_{i}\right]$, and let $T_{i}$ be the integral closure of $L_{i}$ in $F$. Since the condition, $v\left(x_{i}\right)=v(M)$, implies that $R_{v} \supseteq L_{i}$ it follows that $T_{i}=\bigcap\left\{R_{v} ; v \in \Omega, v\left(x_{i}\right)=v(M)\right\}$, and $L=\cap T_{i}$. If $x \in Q_{n}$, then $x$ satisfies an equation, $x^{t}+a_{1} x^{t-1}+\cdots+a_{t}=0$, $a_{i} \in X^{n i}$, so that $x / x_{j}^{n} \in T_{j}, j=1,2, \cdots, r$, and hence $Q_{n} \subseteq x_{j}^{n} T_{j} \cap L$. The minimal property of the $a$-basis $\left\{x_{1}, x_{2}, \cdots, x_{r}\right\}$ assures us that $x_{j}$ is not a unit in $T_{j}$, and since $T_{j}$ is integral over $L_{j}$ there exists at least one real discrete valuation $v$ such that $R_{v} \supseteq T_{j}$ and $v\left(x_{j}\right)>0$. Hence no element different from zero in $T_{j}$ is divisible by all powers of $x_{j}$ so that we have

$$
\bigcap_{n=1}^{\infty} Q_{n}=(0) .
$$

We now assume that $L$ is complete and that its characteristic coincides with that of its residue field. By a theorem of Cohen [2], $L$ then contains a coefficient field $k$, and the power series ring

$$
P=k\left\{x_{1}, x_{2}, \cdots, x_{r}\right\}
$$

is a subring of $L$ over which $L$ is a finite module. If

$$
P_{j}=P\left[x_{1} / x_{j}, x_{2} / x_{j}, \cdots, x_{r} / x_{j}\right],
$$

then $T_{j}$ is integral over $P_{j}$ and the relation between the rings $P$ and $P_{j}$ is similar to the relation between $L$ and $T_{j}$. If $X_{0}=\sum P x_{i}$, and if $\theta \in X_{0}^{n}$, then $\theta / x_{j}^{n} \in P_{j}$. On the other hand if $\pi \in P_{j}$ then $\pi$ is a polynomial in the quotients $x_{i} / x_{j}$ so that if $n$ is its total degree, $x_{j}^{n} \pi$ is a form of degree $n$ in $x_{1}, x_{2}, \cdots, x_{r}$ with coefficients in $P$. Thus $x_{j}^{n} \pi \in X_{0}^{n}$. Moreover, since $P$ is a regular local ring the equation, $X_{0}^{n}:\left(x_{j}^{t}\right)=X_{0}^{n-t}$, holds for all $n \geqq t$, so that if $e$ is the least integer such that $x_{j}^{b} \pi \in P$, then $x_{j}^{e} \pi \in X_{0}^{b}$. This integer $e$ will be called the degree of $\pi$. If $y$ is an element of the quotient field of $P$ that is integral over $P_{j}$, there is an integer $e$ such that $x_{j}^{e} y \in\left(X_{0}^{e}\right)_{b}$. In fact, if $a_{i}$ is the coefficient of $y^{t-i}$ in the equation of integral dependence and if $e_{i}$ is the degree of $a_{i}$, we can take $e$ to be the least integer $f$ such that 
$f i \geqq e_{i}, i=1,2, \cdots, t$. However, the regularity of $P$ implies that the pseudo-valuation associated with the sequence of powers of $X_{0}$ is a valuation and that $X_{0}^{n}$ is a complete ideal for all positive integers $n$. Hence $x_{j}^{c} y \in X_{0}^{b}$ and $y \in P_{j}$. Thus $P_{j}$ is integrally closed.

Lemma 1. If $\theta \in Q_{n}$ and if $g(Z)=Z^{t}+a_{1} Z^{t-1}+\cdots+a_{t}$ is the minimal polynomial for $\theta$ over the quotient field $E$ of $P$, then $a_{i} \in X_{0}^{n i}$.

Proof. As mentioned above, $\theta / x_{j}^{n} \in T_{j}$ and hence $\theta / x_{j}^{n}$ is integral over $P_{j}$. If $h(Z)=Z^{t}+b_{1} Z^{t-1}+\cdots+b_{t}$ is the minimal polynomial of $\theta / x_{j}^{n}$ over $E$, then by a well known lemma of Kronecker [3], $b_{i} \in P_{j}, i=1,2, \cdots, t$. For the same reason, $a_{i} \in P$. Hence since $a_{i}=b_{i} x_{j}^{n i}$, it follows that $a_{i} \in X_{0}^{n i}$, q.e.d.

Lemma 2. If $\theta \in T_{j}$ then there exist integers $s$ such that $x_{j}^{s} \theta \in L$. For any such integer $s$ it is true that $x_{j}^{s} \theta \in Q_{s}$. Moreover, for all positive integers $n, x_{j}^{n} T_{j} \cap L=Q_{n}$.

Proof. As above, the coefficients $a_{i}$ of the minimal polynomial $g(Z)$ of $\theta$ are in $P_{j}$, so that if $d_{i}$ is the degree of $a_{i}$ and $s$ is an integer such that $s i \geqq d_{i}, i=1,2, \cdots, t$ then the minimal equation of $x_{j}^{s} \theta$ has coefficients $x_{j}^{s i} a_{i}$ in $P$, so that $x_{j}^{s} \theta \in L$. On the other hand if $s$ is such that $x_{j}^{s} \theta \in L$, then $x_{j}^{s i} a_{i} \in P$ and hence $x_{j}^{s t} a_{i} \in X_{0}^{s t}$. Thus $x_{j}^{s} \theta$ depends integrally on $X^{s}$ so that $x_{j}^{s} \theta \in Q_{s}$. In particular, for any positive integer $n$ the inclusion, $x_{j}^{n} T_{j} \cap L \subseteq Q_{n}$, holds, and this establishes equality since the opposite inclusion has already been noted, q.e.d.

Proposition 1. Let $(L, M)$ be an integrally closed local domain of the same characteristic as its residue field which is such that its completion $(\bar{L}, \bar{M})$ is also an integrally closed domain. Let $\bar{k}$ be a coefficient field of $\bar{L}$ and let $k=\bar{k} \cap L$. If $\phi\left(Z_{1}, Z_{2}, \cdots, Z_{r}\right)$ is a nonzero form of degree $s$ in the indeterminates $Z$ with coefficients in $k$, then for each positive integer $n \geqq s$, the equality,

$$
Q_{n}:\left(\phi\left(x_{1}, x_{2}, \cdots, x_{r}\right)\right)=Q_{n-s},
$$

holds.

Proof. If $\bar{Q}_{n}=\left(\bar{M}^{n}\right)_{a}$ it follows immediately that $\bar{Q}_{n}=\bar{L} Q_{n}$, so that it is sufficient to prove the proposition under the assumption that $L$ is complete. Assume that $\phi$ is as described and that $\phi(x) \theta \in Q_{n}$. If $g(Z)=Z^{t}+a_{1} Z^{t-1}+\cdots+a_{t}$ is the minimal polynomial for $\theta$ over the ring $P$, then $Z^{t}+\phi a_{1} Z^{t-1}+\cdots+\phi^{t} a_{t}$ is the minimal polynomial of $\phi(x) \theta$ over $P$, and by Lemma $1,(\phi(x))^{i} a_{i} \in X_{0}^{n t}$. Since $P$ is a regular local ring, it follows that $a_{i} \in X_{0}^{n i-s t}$ so that $\theta \in Q_{n-s,}$ q.e.d. 
3. Let $L$ be a two dimensional local domain that is integrally closed in its quotient field $F$, and let the notations of the previous sections be retained.

LEMma 3. If $g$ and $h$ are positive integers, then

$$
\left(X^{\rho} Q_{h}\right):\left(L x_{j}\right)=X^{\sigma-1} Q_{h}, \quad j=1,2 .
$$

Proof. If $x_{1} \theta \in X^{\circ} Q_{h}$, then

$$
x_{1} \theta=\alpha_{0} x_{1}^{0}+\alpha_{1} x_{1}^{o-1} x_{2}+\cdots+\alpha_{0} x_{2}^{o},
$$

where $\alpha_{i} \in Q_{h}, i=0,1, \cdots, g$. Since $L$ is integrally closed, the principal ideal $L x_{1}$ has no embedded components and hence $x_{2}$ is in no prime ideal of $L x_{1}$. Hence equation (2) implies that $\alpha_{\theta}=x_{1} \beta$, and by Proposition 1, $\beta \in Q_{h-1}, x_{2} \beta \in Q_{h}$. Hence we can replace the term $\alpha_{\theta} x_{2}^{o}$ in (2) by $x_{1} \beta x_{2}^{o}$ to obtain $\theta \in X^{o-1} Q_{h}$, q.e.d.

We now formulate two conditions either of which will insure the finiteness of the integral closure of a ring which occurs in the proof of Proposition 2. The first of these is similar to a condition imposed by Rees in [6]. An integrally closed local domain $L$ will be said to satisfy condition $f_{1}$ in case

(a) L has the same characteristic as its residue field;

(b) The completion $\bar{L}$ of $L$ is an integral domain with quotient field $\bar{F}$;

(c) The integral closure $T$ in $\bar{F}$ of any finite ring extension $S$ of $\bar{L}$ such that $S \subseteq \bar{F}$ is a finite $S$-module.

An alternative requirement $f_{2}$ would also serve our purpose. In view of (a) and a theorem of Cohen [2], the ring $\bar{L}$ is a finite module over the power series ring $P=k\left\{x_{1}, x_{2}, \cdots, x_{r}\right\}$, where $k$ is a coefficient field for $\bar{L}$ and $x_{1}, x_{2}, \cdots, x_{r}$ are parameters. Hence the quotient field $\bar{F}$ of $\bar{L}$ is a finite algebraic extension of the quotient field $E$ of $P$. We shall say that $L$ satisfies condition $f_{2}$ if (a) and (b) hold and if $\bar{F}$ is separable over $E$.

Proposition 2. If $(L, M)$ is a two dimensional integrally closed local domain that satisfies either condition $f_{1}$ or condition $f_{2}$, and if $X$ is the ideal generated by a minimal a-basis $x_{1}, x_{2}$ of $M$, then there exists an integer s such that $Q_{s+t}=X^{t} Q_{s}$ for all positive integers $t$.

Proof. It is clearly sufficient to prove the proposition under the assumption that $L$ is itself complete. If $T_{j}, L_{j}$ and $P_{j}(j=1,2)$ are as defined in $\S 2$, then condition $f_{1}$ asserts that $T_{j}$ is a finite $L_{j}$-module. This conclusion can also be deduced from condition $f_{2}$. In fact, $L_{j}$ is integral over $P_{j}$ so that $T_{j}$ is the integral closure in $F$ (the quotient field of the complete domain $L$ ) of the noetherian domain $P_{j}$, and 
since $F$ is separable over $E$ the conclusion follows from the fact that $P_{j}$ is integrally closed.

Let $\omega_{1}, \omega_{2}, \cdots, \omega_{m}$ be a basis for $T_{1}$ as an $L_{1}$ module. By Lemma 2 there is an integer $s$ such that $x_{1}^{s} \omega_{i} \in L, i=1,2, \cdots, m$, and for such an integer $s, x_{1}^{s} \omega_{i} \in Q_{s}$. Now assume that $\theta \in Q_{n}, n \geqq s$, so that $\theta / x_{1}^{n} \in T_{1}$. There are elements $u_{1}, u_{2}, \cdots, u_{m}$ of $L_{1}$ such that

$$
\theta / x_{1}^{n}=u_{1} \omega_{1}+u_{2} \omega_{2}+\cdots+u_{m} \omega_{m} .
$$

Since $u_{i}$ is a polynomial in $x_{2} / x_{1}$ with coefficients in $L$ it follows that there is an integer $g$ such that $x_{1}^{0} u_{i} \in X^{o}, i=1,2, \cdots, m$. Hence if $\mu=\max (s+g, n)$ we can multiply equation (3) by $x_{1}^{\mu}$ to get $x_{1}^{\mu-n} \theta$ $\in X^{\mu-s} Q_{s}$. If $\mu=n$, then $\theta \in X^{n-s} Q_{s}$, and the proof is complete. Otherwise, $\mu=s+g$ and we have $x_{1}^{s+\theta-n} \theta \in X^{0} Q_{s}$, so that by repeated application of Lemma 3 we again find $\theta \in X^{n-s} Q_{s}$, q.e.d.

CoRollaRy. For all integers $a, b \geqq s, Q_{a} Q_{b}=Q_{a+b}$. In particular, $Q_{s}^{n}=Q_{n s}$ for all positive integers $n$.

PROOF. $Q_{a+b} \supseteq Q_{a} Q_{b} \supseteq X^{a+b-2 s} Q_{s}^{2} \supseteq X^{a+b-s} Q_{s}=Q_{a+b}$.

Proposition 3. Under the same hypothesis as Proposition 2, there is an integer $n_{0}$ such that when $n \geqq n_{0}$ the length $\lambda\left(Q_{n}\right)$ of $Q_{n}$ is given by a polynomial in $n$ of degree two that has the same leading term as the Samuel polynomial $\sigma(n)$ that gives the length of $X^{n}$ when $n$ is large.

Proof. It is known [1] that there exist integers $\alpha$ and $\beta$ such that for $a \geqq \alpha$ and $b \geqq \beta, \lambda\left(X^{a} Q_{s}^{b}\right)=B(a, b)$, where $B(a, b)$ is a polynomial in $a, b$ of total degree equal to the dimension of $L$. Hence if $n_{0}=s \beta+\alpha$, and if $n \geqq n_{0}$, Proposition 2 implies that $Q_{n}=X^{n-s \beta} Q_{s}^{\beta}$, so that $\lambda\left(Q_{n}\right)$ $=B(n-s \beta, \beta)$. The conclusion concerning the leading coefficients follows from the fact that $X^{n-s} \supseteq Q_{n} \supseteq X^{n}$, so that $\lambda\left(X^{n-s}\right) \leqq \lambda\left(Q_{n}\right)$ $\leqq \lambda\left(X^{n}\right)$, q.e.d. ${ }^{2}$

4. Let $\left(x_{0}, x_{1}, \cdots, x_{m}\right)$ be homogeneous coordinates of a general point of an arithmetically normal surface $v$ defined over an algebraically closed ground field $k$, assume that the point $(1,0, \cdots, 0)$ is on $v$, that $x_{0}, x_{1}, x_{2}$ are algebraically independent over $k$ and that the coordinate ring $R=k\left[x_{0}, x_{1}, \ldots, x_{m}\right]$ is integral over the ring $k\left[x_{0}, x_{1}, x_{2}\right]$. If $y_{i}=x_{i} / x_{0}$, then $\Sigma=k\left(y_{1}, y_{2}, \cdots, y_{m}\right)$ is the field of rational functions on $v$. Let $z_{i j}=x_{i} x_{j}, i, j=0,1, \cdots, m$. The ring $R_{2}=k\left[z_{00}, z_{01}, \cdots, z_{m m}\right]$ is the ring of homogeneous coordinates on a

2 The referee has called attention to the fact that in view of Proposition 2, the graded ring $\Sigma Q_{n} / Q_{n+1}$ (in the sense of [8, Chapter II]) is a finite module over the ring $\Sigma X^{n} / X^{n+1}$, and that Proposition 3 follows from this. 
derived normal model $v_{2}$ of $v$ belonging to the character of homogeneity 2 [9], while the ring $S=k\left[z_{01}, z_{02}, \cdots, z_{m m}\right]$ is the ring of homogeneous coordinates on the surface $\mathcal{U}$ obtained from $v$ by a quadratic transformation with center $P=(1,0, \cdots, 0)$. Since $z_{00}=z_{01} z_{01} / z_{11}$ it follows that $\Sigma\left(z_{00}\right)=\Sigma\left(z_{01}\right)$ so that $R_{2}$ and $S$ have the same quotient field. Hence if $S_{\delta}$ is the ring of homogeneous coordinates on a derived arithmetically normal model $\mathfrak{u}_{\delta}$ of $u$ belonging to the character of homogeneity $\delta$, then $S_{\delta}$ is a subring of $R_{2}$ in view of the fact that $R_{2}$ is integrally closed. An element of $S_{\delta}$ which is homogeneous of degree $n$ in the natural grading of $S_{\delta}$ will be of degree $n \delta$ if regarded as an element of $R_{2}$ and of degree $2 n \delta$ as an element of $R$, so that the space $U(n)$ of forms in $S_{\delta}$ of degree $n$ is a subspace of the space $V(2 n \delta)$ of forms in $R$ of degree $2 n \delta$.

If $\mathfrak{D}=k\left[y_{1}, y_{2}, \cdots, y_{m}\right]$, if $N=\sum 0 y_{i}$ and if $N_{t}$ is the complete ideal $\left(N^{t}\right)_{a}$, then it is clear that $N_{t}=Q_{t} \cap_{0}$, where $Q_{t}$ is the integral closure of $M^{t}$ in the local ring $\left(0_{N}, M\right)$ at the point $P$ of $v$. In particular, $Q_{t}$ and $N_{t}$ have the same length.

LemMa 4. An element $\omega$ of $V(2 n \delta)$ belongs to the space $U(n)$ if and only if $\omega / x_{0}^{2 n \delta}$ is an element of the ideal $N_{n \delta}$.

Proof. If $\omega \in U(n)$ then $\omega$ satisfies an equation of the form $\omega^{s}$ $+a_{1} \omega^{s-1}+\cdots+a_{s}=0$, where $a_{i}$ is an element of $S$ of degree $n \delta i$. It follows that $a_{i} / x_{0}^{2 n o t}$ is an element of $\mathrm{D}$ in which each term is a power product in $y_{1}, y_{2}, \cdots, y_{m}$ of degree not less than $n \delta i$. Hence $\omega / x_{0}^{2 n \delta}$ is integral over $N^{n \delta}$ and is therefore in $N_{n \delta}$.

Assume on the other hand, that $\omega \in V(2 n \delta)$ and that $\theta=\omega / x_{0}^{2 n \delta} \in N_{n \delta}$. The element $\theta$ satisfies an equation of the form $\theta^{t}+b_{1} \theta^{t-1}+\cdots+b_{t}$ $=0$, with $b_{i} \in N^{n \delta i}$. Thus the coefficient $b_{i}$ can be written as a polynomial in $y_{1}, y_{2}, \cdots, y_{m}$ in which no term is of degree less than $n \delta i$. In view of the relations, $z_{00} y_{k}=z_{0 k}, z_{0 j} y_{k}=z_{j k}$, it follows that $z_{00}^{n \delta t} b_{i}$ is an element of $S$. Hence if we multiply the equation for $\theta$ by $z_{00}^{\text {nst }}$ we find an equation that expresses the integral dependence of $\omega$ on $S$. Since $\delta$ is a character of homogeneity, it follows that $\omega$ is an element of $S_{\delta}$ of degree $n$, q.e.d.

Since the dimensions of the spaces $U(n)$ and $V(2 n \delta)$ are given by the Hilbert functions $\chi\left(\mathcal{U}_{\delta}, n\right)$ and $\chi(\mathcal{V}, 2 n \delta)$ respectively, and since $\lambda\left(N_{n}\right)=\lambda\left(Q_{n}\right)$ for all $n$, the equality,

$$
\chi(\mathcal{v}, 2 n \delta)-\chi\left(\mathcal{U}_{\delta}, n\right)=\lambda\left(Q_{n \delta}\right),
$$

is an immediate consequence of Lemma 4.

It should be noted that equation (4) implies that the length $\lambda\left(Q_{n \delta}\right)$ is a polynomial in $n$ when $n$ is large, so that if the local ring $\left(0_{N}, M\right)$ 
satisfies either condition $f_{1}$ or $f_{2}$, this polynomial will coincide with the one described in Proposition 3 with $n$ replaced by $n \delta$. It is not difficult to see that $\left(0_{N}, M\right)$ satisfies condition $f_{2}$. In fact, condition (a) is automatically satisfied and condition (b) is a consequence of the analytical irreducibility of normal varieties. As to the separability requirement imposed by $f_{2}$ we note first that since $k$ is algebraically closed we can assume without loss of generality that the quantities $\left(y_{1}, y_{2}\right)$ form a separating transcendence base for $\Sigma / k$ and at the same time form a minimal $a$-basis for the maximal ideal $M$ in $\mathrm{o}_{N}$. If $(\bar{L}, \bar{M})$ is the completion of $\mathrm{o}_{N}$ relative to the powers of $M$, and if $P=k\left\{y_{1}, y_{2}\right\}$, then $\bar{L}$ is integral over $P$ and it is a straightforward matter to see that every $k$-derivation of $P$ admits a unique extension to a $k$-derivation of $\bar{L}$. Indeed, the term " $k$-derivation" is used here in the sense of $[4, \S 3]$, and such derivations are uniquely determined by their restrictions to dense subrings. Since $\boldsymbol{o}_{N}$ is dense in $\bar{L}$ and the polynomial ring $P_{0}=k\left[y_{1}, y_{2}\right]$ is dense in $P$, and since any $k$-derivation of $P_{0}$ admits a unique extension to $D_{N}$ in view of the separability of $\Sigma$ over $k\left(y_{1}, y_{2}\right)$, our assertion follows. Hence it follows also that the quotient field $F$ of $\bar{L}$ is separable over the quotient field $E$ of $P$ as condition $f_{2}$ requires.

\section{BIBLIOGRAPHY}

1. P. B. Bhattacharya, The Hilbert function of two ideals, Proc. Cambridge Philos. Soc. vol. 53 (1957) pp. 568-575.

2. I. S. Cohen, On the structure and ideal theory of complete local rings, Trans. Amer. Math. Soc. vol. 59 (1946) pp. 54-106.

3. W. Krull, Beiträge zur Arithmetik kommutativer Integritätsbereche, I, Math. Z. vol. 41 (1936) pp. 545-577.

4. Masayoshi Nagata, Note on complete local integrity domains, Mem. Col. Sci. Univ. Kyoto vol. 3 (1954) pp. 271-278.

5. D. G. Northcott and D. Rees, Reductions of ideals in local rings, Proc. Cambridge Philos. Soc. vol. 50 (1954) pp. 145-158.

6. D. Rees, Valuations associated with ideals, Proc. London Math. Soc. vol. 6 (1956) pp. 161-174.

7. M. Sakuma, On prime operations in the theory of ideals, J. Sci. Hiroshima Univ. Ser. A vol. 20, no. 3 (1957) pp. 101-106.

8. P. Samuel, Algèbre locale, Paris, Gauthier-Villars, 1953.

9. O. Zariski, Some results in the arithmetic theory of algebraic varieties, Amer. J. Math. vol. 61 (1939) pp. 249-294.

State University of Iowa 